\title{
Reflets
}

Revue ontaroise d'intervention sociale et communautaire

\section{Le Consortium national de formation en santé}

\section{Gilles Renault, Pierre Gaudet et Rachel Ouellette}

Volume 9, numéro 2, automne 2003

Travail et mieux-être

URI : https://id.erudit.org/iderudit/011100ar

DOI : https://doi.org/10.7202/011100ar

Aller au sommaire du numéro

Éditeur(s)

Reflets : Revue ontaroise d'intervention sociale et communautaire

ISSN

1203-4576 (imprimé)

1712-8498 (numérique)

Découvrir la revue

Citer cet article

Renault, G., Gaudet, P. \& Ouellette, R. (2003). Le Consortium national de formation en santé. Reflets, 9(2), 241-252. https://doi.org/10.7202/011100ar

Tous droits réservés (C) Reflets : Revue ontaroise d'intervention sociale et communautaire, 2002
Ce document est protégé par la loi sur le droit d'auteur. L'utilisation des services d'Érudit (y compris la reproduction) est assujettie à sa politique d'utilisation que vous pouvez consulter en ligne.

https://apropos.erudit.org/fr/usagers/politique-dutilisation/ 


\section{Le Consortium national de formation en santé}

Ce texte a été rédigé par Gilles Renault ${ }^{1}$. Il est construit à partir des documents qui ont servi à la demande de subvention du Consortium national de formation en santé (CNFS) présentée à Santé Canada (juillet 2003). Certains extraits du texte ont aussi été écrits par Pierre Gaudet, directeur général du CNFS et Rachel Ouellette, directrice des opérations du CNFS pour l'Université d'Ottawa.

\section{Introduction}

L'Université Laurentienne est un des membres du Consortium national de formation en santé (CNFS). La Laurentienne œuvre à l'augmentation de l'accessibilité de ses programmes de santé et au développement de nouveaux programmes dans ce domaine. Son objectif est de mieux répondre aux besoins en matière d'éducation de la clientèle du Nord de l'Ontario et des francophones vivant en situation minoritaire à l'extérieur du Québec.

Au sein du CNFS, on trouve les institutions d'éducation postsecondaire suivantes: l'Université Laurentienne, l'Université d'Ottawa, l'Université de Moncton, l'Université Saint-Jean, le Collège universitaire Saint Boniface, la Faculté Saint-Jean de l'Université de l'Alberta, le Collège communautaire de Campbelton, la Cité collégiale, le Collège Boréal. Il est aussi actif dans l'Entente Québec/Nouveau Brunswick. Toutes ces institutions offrent des programmes en français dans différentes disciplines de la santé. 
Le présent texte offre un aperçu de ce que le CNFS veut entreprendre au cours des cinq prochaines années en mettant un accent particulier sur l'Université Laurentienne.

\section{La Mission du Consortium national de formation en santé}

La mission du CNFS est d'assurer la mise en œuvre d'un réseau postsecondaire de formation et de recherche en français. Par cette mission, le CNFS appuie les institutions de formation qui offrent ou qui pourraient offrir une formation dans le domaine de la santé, les chercheurs dans le domaine de la santé et, plus particulièrement, dans le domaine de la santé des communautés francophones en situation minoritaire.

Le CNFS souhaite répondre aux besoins en matière de santé des communautés francophones en situation minoritaire et contribuer de façon significative à leur mieux-être et leur plein épanouissement. Pour ce faire, le CNFS s'est fixé les objectifs suivants:

- connaître les besoins spécifiques des communautés et favoriser l'insertion des professionnels formés dans leur lieu de provenance;

- maximiser l'apport des institutions existantes en déployant leurs capacités de formation;

- favoriser l'accès à de nouvelles formations dans les communautés qui en sont dépourvues;

- favoriser les partenariats et les collaborations;

- faciliter et entretenir la liaison et la concertation au sein du réseau ainsi qu'avec les réseaux complémentaires. 


\section{Projet de formation et de recherche 2003-2008 - CNFS - Université Laurentienne}

L'Université Laurentienne offre une panoplie de programmes en santé. Elle s'est donc joint aux autres institutions d'enseignement du CNFS afin d'offrir d'autres programmes qui ne sont pas disponibles en son sein, ou pour faire la promotion des programmes offerts ailleurs. Comme pour ses partenaires du CNFS, le «Projet de formation et recherche, 2003-2008", lui permettra d'assurer la continuité des programmes établis et d'élargir sa programmation pour mieux répondre aux besoins.

Le Projet de formation et de recherche est une proposition collective et concertée. Il présente dix projets provenant des institutions en plus de celui du Secrétariat national qui effectue la coordination d'ensemble du CNFS. Le Projet vise un déploiement de la formation en santé dans l'ensemble des institutions et dans les régions et les communautés francophones. Il favorise l'augmentation des ressources francophones dans le domaine de la santé, et que la provenance des étudiantes et des étudiantes viennent des communautés mêmes et qu'ils y retournent pour pratiquer leur profession.

L'Université Laurentienne et ses partenaires du CNFS, ont décidé d'articuler le projet de développement de la formation postsecondaire dans le domaine de la santé autour de quatre grands axes:

1. le recrutement;

2. la formation;

3. la recherche;

4. la gestion et la coordination du projet. 


\section{Les axes de développement spécifiques à l’Université Laurentienne}

\section{Le recrutement}

À l'Université Laurentienne, l'axe du recrutement poursuit les objectifs suivants:

- développer une planification stratégique de recrutement en liaison avec les autres instances de recrutement sur campus, en collaboration avec les partenaires du Consortium et en collaboration avec le gouvernement provincial et ses coordonnatrices du ministère de la santé et,

- développer le matériel promotionnel pour les programmes impliqués dans le projet.

Le recrutement des étudiants, qui souhaitent poursuivre une carrière en santé dans la francophonie hors Québec, constitue un premier axe d'intervention dans le cadre d'une perspective d'accroissement des professionnels de la santé. Tout d'abord, il s'agit de développer l'intérêt des jeunes dans le domaine de la santé et, ensuite, les aider à s'orienter vers les possibilités d'études en français dans les établissements de leur communauté.

Dans la phase II, il s'agira de continuer à accorder une grande place à un recrutement actif et inventif, et de poursuivre et d'élargir à l'ensemble du réseau des communautés les mesures entreprises ou envisagées de façon encore limitée.

Le recrutement étudiant doit être complété par des mesures visant le recrutement professoral afin de briser le mythe qu'on enseigne peu dans le domaine de la santé en français, faute d'enseignants et de chercheurs disponibles. Ces mesures d'appui visent non seulement le recrutement habituel de professeurs, mais elles favoriseront la sélection et la rétention d'étudiants de deuxième cycle prometteurs pour l'enseignement et la recherche. Bref, la relève nécessaire en milieu francophone minoritaire. 


\section{La formation}

À l'Université Laurentienne, l'axe de la formation poursuit l'objectif principal d'augmenter le nombre d'étudiantes et d'étudiants. Pour ce faire, il lui faut:

- développer les capacités de la formation pratique complémentaire;

- accroitre le potentiel de formation à distance et les partenariats pour la livraison des cours;

- développer de nouveaux partenariats pour partager et rendre accessible la formation et les ressources dans l'ensemble du réseau;

- développer une programmation pour le développement professionnel (la formation continue) des infirmières, des travailleurs sociaux et des autres professionnels de la santé.

Ici, l'axe central du projet reste la formation qui comprend l'enseignement théorique ou en salle de classe et la formation clinique ou pratique dans le milieu même des services de santé.

Cependant, comme plusieurs programmes ne seront pas disponibles dans l'ensemble des différents établissements et dans plusieurs régions, c'est ici que les expériences de partenariat en développement, pour des enseignements partagés ou complémentaires, permettent d'envisager la mobilité et la dissémination des enseignements selon diverses formules. C'est pourquoi, dans la phase I, on a donné une place importante à la formation à distance et à des activités de médiatisation de l'enseignement. Ces activités permettent une plus grande accessibilité. Toutefois, il s'agit d'une dimension complexe qui implique non seulement la transposition des contenus de cours et une maitrise des technologies appropriées, mais le développement d'une nouvelle forme de pédagogie dans laquelle les corps enseignant et étudiant doivent investir.

La formation clinique ou pratique est une dimension importante des disciplines de la santé. Elle est aussi la dimension qui rattache le professionnel en devenir avec le milieu dont il provient. Il s'agit pour la ou le futur professionnel d'une façon privilégiée 
de mettre en application sa formation théorique dans le contexte communautaire qui pourra alors bénéficier de ses services. De son côté,l'étudiant pourra mieux connaitre ce milieu professionnel et s'y insérer.

La description de la formation est un objectif à atteindre dont la réalisation n'est pas simple. Au contraire, il faut des places, des précepteurs, des agréments universitaires et professionnels, un appui aux étudiantes et étudiants, tout particulièrement lorsqu'il vient d'une région éloignée. Ces types d'encadrements sont à développer dans plusieurs disciplines et divers milieux, ce qui exige du temps, des ressources humaines, de nombreux échanges et une bonne dose de collaboration.

En médecine, la situation est complexe. Les milieux de stages en milieu minoritaire francophone sont limités pendant le cours des études de premier cycle. La situation est encore plus difficile lorsque les étudiants atteignent le deuxième cycle et qu'ils doivent obtenir des résidences. Des professions aussi spécialisées que celles du domaine de la santé, associées à la recherche de fine pointe et aux nouvelles possibilités technologiques, font appel à la formation continue. Par conséquent, un réseau de formation peut être d'un grand appui car il permet le développement et rend largement disponibles des expériences et des ressources en ce sens.

\section{La recherche}

À l'Université Laurentienne, l'axe de la recherche a comme objectif de contribuer au développement de la recherche en santé visant les communautés francophones minoritaires. Pour ce faire, le projet doit intégrer la dimension de la recherche au cœur même d'un enseignement universitaire d'avenir. Il doit aussi l'insérer au cœur de la problématique de la santé de la francophonie hors Québec en ce sens que la spécificité de ses besoins et la façon d'y répondre restent à explorer, à documenter, à rassembler.

Le Projet ne joue par un rôle d'organisme subventionnaire mais, plutôt, il est un organisme de facilitation, de concertation et d'appui aux chercheures et chercheurs et de sensibilisation auprès 
des organismes subventionnaires, le cas échéant. Dans ce contexte, les types d'actions à appuyer seraient principalement:

- la formation de chercheures et de chercheurs et la formation de la relève de professeurs du même coup, compte tenu de la pénurie dans plusieurs domaines de la santé;

- des activités de réseautage et d'échanges, incluant des forums, des conférences, des visites entre les chercheurs des différentes institutions et des milieux cliniques des communautés francophones minoritaires;

- la formation d'équipes pluridisciplinaires et interinstitutionnelles autour de thèmes prioritaires et favorisant des regroupements;

- des activités d'appui pour la préparation de demandes et la sensibilisation des pourvoyeurs de fonds.

\section{La gestion et la coordination du projet}

À l'Université Laurentienne, l'axe de la gestion et de la coordination du projet a comme objectif de contribuer aux activités de coordination et de réseautage au niveau local, régional, provincial et national.

\section{L'évaluation du projet}

Afin d'évaluer adéquatement le projet, l'Université Laurentienne assure, annuellement, la collecte, l'analyse et l'évaluation des données relatives aux résultats obtenus en fonction des objectifs fixés et des indicateurs de rendement présentés auparavant. Elle fera ensuite état des résultats dans un rapport d'activités annuel transmis à Santé Canada et au Secrétariat national du CNFS, lequel, sur cette base, proposera l'évaluation globale au Conseil d'administration du Consortium, ainsi qu'à Santé Canada.

De façon complémentaire, au cours de la troisième année du Projet de formation et de recherche (2005-2006) et de la dernière année du projet (2007-2008), le CNFS fera appel à un consultant 
externe afin de procéder à une évaluation globale. Des données supplémentaires seront alors recueillies auprès des partenaires des milieux cliniques, des bailleurs de fonds, des étudiants, des membres du personnel, des représentants des réseaux de santé dans les communautés et de toutes les autres personnes ou groupes associés au projet. Effectuées en collaboration avec les partenaires, les évaluations permettront d'apporter les ajustements nécessaires au projet afin d'assurer l'atteinte des objectifs fixés et des résultats escomptés. L'intégration des résultats de ces évaluations sera réalisée via la mise à jour régulière des plans de travail qui guideront les activités du projet.

\section{Visées du projet de formation et de recherche}

On aura compris que pour l'Université Laurentienne, ce projet de formation et de recherche a certaines visées. En effet, par ce projet, on rendra possible la mise sur pied, à partir d'avril 2003, de programmes et d'activités afin d'améliorer l'accès à des services de santé en français dans les communautés francophones en situation minoritaire par la disponibilité d'un plus grand nombre de professionnels francophones de la santé provenant de ces communautés. De plus, pour l'Université Laurentienne, on vise:

- une augmentation du nombre d'étudiants dans les programmes de la santé (254 pour l'Université Laurentienne, 2500 pour l'ensemble du CNFS et une diplômation d'environ 1200 professionnels de la santé);

- le développement des capacités de la formation clinique et pratique;

- l'accroissement du potentiel de la formation à distance et des partenariats pour sa livraison dans cette grande région;

- le développement de nouveaux partenariats pour faciliter l'accessibilité des programmes offerts dans l'ensemble du réseau;

- le développement d'une programmation de développement professionnel conjointement avec les associations et les ordres professionnels; 
- la contribution au développement de la recherche en santé visant les communautés francophones minoritaires;

- la contribution aux activités de coordination et de réseautage.

Ainsi, l'Université Laurentienne prévoit une augmentation du nombre d'étudiants au sein des programmes d'études en santé d'ici l'an 2007-2008. L'accessibilité à certains programmes existants pourra être accrue et le développement de nouveaux programmes permettra de garder les professionnels dans leur milieu de provenance, principalement dans le Nord ontarien, comme dans les régions hors province. Plusieurs consultations ont montré que les jeunes allant étudier ailleurs ne revenaient pas dans leur milieu. L'exode de nos professionnels de la santé s'accroît rapidement. Statistiques Canada montre clairement que la population du Nord de l'Ontario diminue à un rythme accéléré, plus vite que le reste de la province.

L'Université Laurentienne en favorisant une approche régionale de collaboration entre les établissements d'enseignement postsecondaires du Nord de l'Ontario et les organismes communautaires, donne la possibilité de faire sa formation clinique et pratique dans sa région. La force de l'Université Laurentienne réside dans son réseau établi avec sa formation à distance qui existe depuis déjà plusieurs années. Le réseau Contact Nord facilite également la mise sur pied de lieux de formation encadrés. L'Université Laurentienne prévoit offrir une programmation médiatisée en sciences infirmières, en travail social, en éducation physique (promotion de la santé) et en médecine, entre autres. Le modèle prévoit la mise sur pied d'une équipe de médiatisation qui a comme objectif d'encadrer les professeurs dans le développement de cours médiatisés et d'offrir le soutien technologique pour la conception et pour la livraison. Les professeurs impliqués dans la médiatisation des cours combinent le cours disponible sur campus et le cours médiatisé. Cette approche permet d'établir un lien entre le programme sur campus et celui «en ligne». À cet égard, l'École de service social sera le premier programme à explorer et à vérifier la portée du modèle. 
L'Université Laurentienne en partenariat avec l'Université Lakehead de Thunder Bay a obtenu l'approbation des instances gouvernementales ontariennes pour mettre en place une École de médecine. L'École de médecine du Nord de l'Ontario (EMNO) offrira, dans un premier temps, une programmation en anglais, le financement actuel étant pour une programmation anglophone. Toutefois, le CNFS, en injectant des fonds supplémentaires, pourra offrir aux étudiants francophones la possibilité d'étudier en français, du moins, obtenir leur formation clinique près de leur lieu de provenance.

L'Université Laurentienne développe une approche permettant un apprentissage autonome, l'étudiant demeurant dans son milieu et progressant à son rythme tout en ayant recours à certaines personnes ressources de son milieu qui l'accompagneront dans sa démarche. L'Université entend aussi utiliser la communauté en tant que communauté de savoir, les professionnels sur place agissant à titre de facilitateurs ou comme chargés de cours.

Afin de répondre adéquatement à la réalité, une approche régionale ou approche coordonnée, entre les établissements postsecondaires dans le Nord ontarien (le Collège Boréal, l'Université Laurentienne, le Collège universitaire de Hearst et l'Université de Sudbury), s'avère nécessaire et incontournable. Cette collaboration, qui existe déjà pour certains programmes et qui est amorcée pour d'autres, permettra d'élargir l'accessibilité à des programmes existants et de développer de nouveaux programmes. La mission de ces établissements postsecondaires étant différente et distincte, cette approche régionale coordonnée doit respecter l'autonomie de chacun.

L'Université Laurentienne facilitera le développement professionnel dans le domaine de la santé. L'Université offre déjà une série de cours en soins cardiaques pour les infirmières autorisées et une série de séminaires sur la supervision en travail social.Étant consciente des distances séparant la région des grands Centres de formation du Sud et de l'Est de l'Ontario, l'Université veut développer des structures qui permettront à ces professionnels d'améliorer leur formation professionnelle dans leur lieu d'intervention. L'Université Laurentienne, avec son approche régionale 
facilitera les liens entre les différents chercheurs et chercheures en santé dans le Nord de l'Ontario. En effet, une structure développée en collaboration avec les établissements d'enseignement postsecondaire permettra une liaison plus étroite entre les chercheurs. Des rencontres et des colloques annuels favoriseront également les échanges entre les chercheures et chercheurs et les intervenantes et intervenants.

\section{Conclusion}

Le Gouvernement du Canada a montré sa volonté de contribuer à l'amélioration de l'accès des francophones en situation minoritaire à des services de santé dans leur langue. Il l'a fait en appuyant, de 1999 à 2003, un premier projet de formation accrue: celui du Centre national de formation en santé, coordonné par l'Université d'Ottawa, en collaboration étroite avec l'Hôpital Montfort dont la survie était menacée. Il l'a aussi fait en visant le développement des partenariats entre les institutions postsecondaires de la francophonie canadienne. L'engagement du gouvernement fédéral s'est également concrétisé du côté du Ministère de la santé du Canada, qui formait, au printemps 2000, un Comité consultatif des communautés francophones en situation minoritaire (CCCFSM) pour le conseiller sur la situation des soins de santé en français dans la francophonie minoritaire canadienne.

Une première étude, publiée en juin 2001 par la FCFA, pour le compte du CCCFSM de Santé Canada, et intitulée Santé en français: pour un meilleur accès à des services de santé en français, brosse le portrait des besoins criants des communautés francophones minoritaires en matière de santé. L'étude permet aussi de constater qu'en moyenne, plus de la moitié des francophones qui vivent en situation minoritaire n'ont pas ou ont rarement accès à des services de santé dans leur langue. Même si des francophones de certaines régions disposent d'un meilleur accès aux services de santé en français, l'étude indique que des améliorations sont nécessaires 
dans toutes les régions. L'étude met aussi en relief l'importance de la langue maternelle dans la qualité et l'efficacité du nombre de soins fournis, signalant qu'elle constitue aussi un facteur thérapeutique.

Pour pallier aux lacunes, la formation apparait indispensable d'autant plus que le nombre de professionnels pouvant offrir des services en français et le taux d'inscriptions dans les programmes de formation en santé sont actuellement nettement insuffisants. À partir de ces études, on estime qu'il faudrait tripler, voire même quadrupler le nombre d'inscriptions de francophones dans des programmes de formation en santé pour répondre aux besoins actuels des populations de la francophonie minoritaire au Canada.

La nature même des programmes postsecondaires en santé pose cependant des défis. Il s'agit, dans la plupart des cas, de formations exigeantes, complexes et spécialisées, pour lesquelles les enseignantes et enseignants se font rares et où les étudiantes et étudiants doivent nécessairement se déplacer pour poursuivre leur formation. Or, une fois déracinés, peu d'entre eux retournent exercer leur nouvelle profession dans leur communauté d'origine. Il importe donc de développer davantage la formation en français des étudiantes et des étudiants afin qu'ils puissent la faire dans leur milieu d'origine et qu'ils puissent exercer leur profession dans leur communauté et dans leur langue maternelle. En ce sens, la présente position de l'Université Laurentienne et de ses partenaires du CNFS s'inscrit parfaitement dans la stratégie mise de l'avant par le projet des langues officielles en contexte minoritaire du ministre Dion.

\section{Notes}

1. Au moment de la rédaction de ce texte par Gilles Renault, celui-ci était coordonnateur du CNFS-Université Laurentienne. 